\title{
IoT Middleware Architecture based on Ontologies to Model Logistic Process
}

\author{
S. Aoulad Allouch, K. Amechnoue, I. Achatbi \\ National School of Applied Sciences Tangier \\ saloua.aoulad@gmail.com; kamechnoue@gmail.com;i.achatbi@gmail.com
}

\begin{abstract}
In recent years, the Internet of Things (IOT) become a promising topic of technical social and economic significance, especially with the high number of developed sensors and technologies. Logistic applications are a perfect domain of loT as it adds new functionalities in identification, traceability, storage and realtime tracking of good in the supply chain. Handling the huge quantities of heterogeneous loT components and logistic items is an important challenge. We present in this paper a new Internet of Things middleware architecture for logistic transport applications. This architecture focuses mainly on a semantic model that uses ontologies for sensor data representation by describing the main entities involved in the logistic scenario.
\end{abstract}

Keywords-component; loT; Middleware; semantic and ontology; supply chain; logistic

\section{Introduction}

IoT can be traced back to the pioneering work done by Kevin Ashton from Massachusetts Institute of Technology (MIT), Auto-ID Center in 1999, and was initially linked to the new idea of using radio frequency identification (RFID) and electronic product code (EPC) in the supply chain [1]. It is envisioned that billions of objects will be equipped with different kinds of sensors, actuators and mobile devices and connected to the internet via heterogeneous access networks.

Logistics is the art of managing, controlling and implementing the flow of goods, services and related information between the point of origin and the point of consumption. In the other hand, logistic has become an important component of economy and more interest are given to this domain. As it is necessary to have professional and intelligent logistical support to achieve international trading and distribution process, loT is mainly applied. In fact, loT will provide intelligence to the logistics distribution network, transparency and real-time to the items management of the logistic system, moreover it can reduce the storage cost and improving the logistics service quality, so that the whole supply chain integrates more closely.

A logistic domain may have a relatively large number of applications and information sources, likewise the loT components are heterogeneous, the number of sensor and actuators involved are growing, so a massive real-time data flow will be produced by this devices. The data provided by different objects, sensing devices and that relating to the logistics domain, must be organized and interpreted in a 
S. Aoulad Allouch, K. Amechnoue, I. Achatbi; IoT Middleware Architecture based on Ontologies to Model Logistic Process. Transactions on Machine Learning and Artificial Intelligence, Vol 5 No 4 August (2017); pp: 85-92

homogeneous way to allow interoperability in the logistics chain, likewise define corresponding events for the different situations of products.

In the light of the challenge above, we propose an loT middleware architecture based on semantic and ontology to describe the sensing devices and their functionalities in loT, thus, to model the logistic domain and facilitate collaboration and interoperability between system components.

The remainder of the paper is organized as follows: Section 2 gives an overview of the background of our work from the vision of middleware, semantic and ontologies. The related work concerning logistic and sensor ontology is briefed in Section 3. Section 4 presents the proposed loT middleware architecture and its main elements. Finally, in section 5 we give a conclusion and we discuss briefly our future work.

\section{Background}

In the context of logistics applications, our goal is to enhance collaboration between stakeholders in logistic chain for an intelligent monitoring, and a better coordination and visibility to respond to customer orders and market demand. This by given more attention to traceability and real-time tracking of goods in the chain. IOT can act as the basis for the development of services and applications that offer information sharing between logistics partners, this, provide improved visibility of supply chain. To model logistic process with the loT, the integration of several technologies is very important and it involves three major categories: Radio-Frequency Identification (RFID), sensors and embedded systems for identifying, sensing and reading logistic items. Due to the large variety of used technologies, loT requires interoperability between devices and information, to address this concern; we propose an loT middleware for logistic applications based on semantic and ontology.

\subsection{Middleware}

The middleware is an interface that facilitates the interaction between technological and the application levels, this with providing technical interoperability. IoT is characterized by a heterogeneous and dynamic infrastructure comprised of an ultra large number of things, so, collecting data from these different objects is an important task as it allows software systems to understand the environment better. Further, we cannot expect all these objects to be connected to the computers due to technical and economic reasons. In this context middleware has a major role in simplifying the development of new services and the integration of legacy technologies into new ones, thus, loT middleware sits between the loT hardware and data and the applications that developers create to exploit the loT.

\subsection{Semantic Web and Ontologies}

The term "Semantic Web" refers to World Wide Web Consortium (W3C) vision for the future of the Web in which information is given well defined meaning, making it simpler for machine to perform more work involved in finding, combining and acting upon information on the web. Thus, enabling computers and people to work in cooperation. The collection of Semantic Web technologies (RDF, OWL, SKOS, SPARQL, etc.) provides an environment for people to create data stores on the web, build vocabularies, and write rules for handling data.

Since the early 1990s, ontologies have become an important research topic examined by numerous research communities, several definitions of ontologies have been suggested in the last decades. But the one that best describes ontologies can be found in Gruber [2] "ontology is a formal, explicit specification 
of a shared conceptualization". Similarly, the W3C[3] defines ontology as the terms used to describe and represent an area of knowledge, and is used by people, databases, and applications that need to share domain information. Ontology has been widely used to facilitate knowledge sharing and reusing. For pervasive computing environments, using ontology to model context and situation enables multiple entities to have common understanding of the context and situation during collaboration. An otology comprises three elements: First, classes in the many domains of interest, second, relationships that can exist among things, third, the properties those things may have.

\section{Related work}

\subsection{Sensor ontology}

Nowadays, most research and standardization related to loT domain have principally concentrated in sensor description and observation data modeling. Thus, researchers have realized that the data sharing and service interoperability between different data models and devices can be handled using semantic web technologies. In addition; they confirm that ontology serves as a key factor to enable interoperability between heterogeneous systems. Several ontologies have been presented for sensor. The work in [4] presents an attempt to capture the most important features of a sensor node that describes its functionality and its current state. The ontology describes the main components of a sensor node such as processor CPU, power supply, and radio and sensor modules. OntoSensor[5] constructs an ontology comprised of definitions of concepts and properties adopted in part from SensorML[6]an extensions to the IEEE Suggested Upper Merged Ontology (Sumo)[7]. However, it does not provide a descriptive model for observation and measurement data.

The W3C Incubator Group on Semantic Sensor Networks (SSN) has introduced ontology[8] to describe sensors and sensor networks. The ontology represents a high-level schema model to describe sensor devices, their capabilities, platform and other related attributes in the semantic sensor networks and the sensor Web applications. Nevertheless, SSN ontology does not include units of measurement, location, hierarchies of sensor types and domain knowledge that are related to sensor data. Thus, concepts were included to allow linking to such external ontologies.

Semantic Sensor Observation Service ontology (SemSOS or O\&M-OWL) [9] models sensor observations and measurement in OWL, it provides the ability to query high-level knowledge of the environment as well as low-level raw sensor data, besides it adds semantics essentially to weather domain.[10]has proposed an ontology that models three aspects of the real world present in the loT; things, real world concepts and functionalities of things and real world approximation, and this by linking, the domain of knowledge for sensing, actuating, and processing tasks and the real world representation through loT services that are aware of their environment. The principal idea of this ontology is that it creates a direct relation between physical concept, mathematical formulas and functions.

\subsection{Logistic ontology}

Many initiatives have been taken by researcher in the domain of logistic ontology:

[11]Proposed a logistic ontology based on situations semantic by identifying a set of logistic events and logistics situations founded on logistic process that model the transition of product. Thus, they use a situation model based on semantic to specify a situation of product and determine events caused by this situation. [12]Contribute in the advancement of logistic ontologies by referring to supply chain 
S. Aoulad Allouch, K. Amechnoue, I. Achatbi; IoT Middleware Architecture based on Ontologies to Model Logistic

Process. Transactions on Machine Learning and Artificial Intelligence, Vol 5 No 4 August (2017); pp: 85-92

management and reusing the existing body of knowledge contained in the SCOR (Supply Chain Operation Reference) model. As the SCOR model does not aim at covering interorganizational logistics to the full extend, this ontologies lack some characteristic concepts of this domain.

In another tentative,[13]build a logistic domain ontology model (LDOM) that represent relations among logistic domain knowledge. This ontology contains 12 top classes - namely cargo, organization, supplier, customer, carrier, transport service, constraint, transport service standard, transport mode, vehicle, traffic line and information between actors. [14]Proposes a core ontology that determines the principal concepts usually adopted in the logistics domain, thus it can be used as the basis to promote communication, facilitate integration of heterogeneous IT system between organizations, and finally it is used for engineering purposes.

\section{Global Overview of the Proposed Architecture}

\subsection{Global IoT architecture for logistics}

The proposed loT logistic architecture presented in Fig, adopts a service oriented approach as generally done in the literature, based especially on semantics to describe devices, their data, and their physical attributes. The adoption of the SOA principles allows for decomposing complex and monolithic systems into applications consisting of an ecosystem of simpler and well-defined components [15][16],thus, it is divided into four layer: perception layer, network layer, semantic middleware layer and application layer as designed in Figure 1.

Perception layer:

The focal mission of this layer is to perceive the physical properties of things during the logistic process. This information is about state of good and vehicles or their environment such as: temperature, humidity, orientation, chemical changes in the air... etc. This process of perception is based on several sensing technologies (e.g. Sensor, RFID, NFC, GPS, etc.).Hereafter, the collected information are converted to digital signal and then passed to Network layer for their transmission.

Network layer:

This layer is mainly responsible for the transmission of information collected in perception layer to middleware layer. The transmission medium can be wired or wireless and technology can be 3G, UMTS, $\mathrm{Wi}-\mathrm{Fi}$, ZigBee, Bluetooth etc. depending upon the sensor devices.

Semantic Middleware layer:

This layer sits between applications, and the network layer and helps brings together a multitude of devices and data in a way that permits developers to create a new loT services without having to know the detail of each device or data format.

Our future loT middleware is an extended of the work done in [17], it involves four important parts: a discovery module, service management, service composition and a knowledge base.

Discovery module consists of two parts: device discovery which enables any device in the loT network to detect all its neighboring devices and make its presence known to each neighbor in the network. Service discovery: is used to find appropriate devices according to the service description submitted by the requestor. 
Service management provides the main functions that are expected to be available for each objects and that allow for their management in the loT scenario.

Service composition gives the functionalities for the composition of single service offered by networked objects to build specific applications; in this component we find the process responsible for coordinating all tasks.

Knowledge Base is the main component of this middleware; it contains all information needed by other piece, and gives a comprehensive set of ontologies that describe logistic items, logistic process, sensor, actuator and all physical concept and unit of measurement needed in our case.

Application layer:

The principal goal of this layer is the management of application based on the objects information handled in the middleware layer, in our case it comprise all functionalities offered to stakeholders in logistic process, as tracking and checking the state of goods and environment, react to changes in task and network requirements, thus, sharing important information with the right actor at the right time, and responding dynamically to the order market demand.

In our work, more attention will be given to the semantic middleware layer especially on modeling a set of ontologies to describe goods, environment and the devices used in the logistic process.

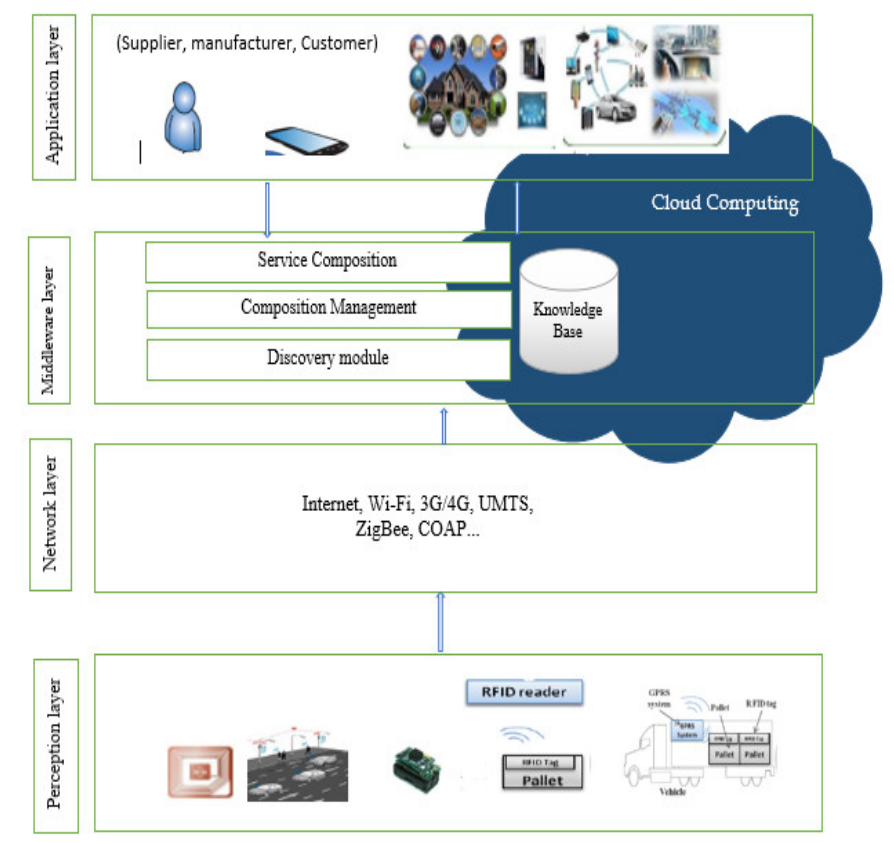

Figure 1: General loT middleware Architecture for logistic process

\subsection{Proposed ontologies}

In this section, we will present a set of ontologies that models device, thing and logistic. Our work is based on the advantages and shortcomings of the ontologies listed above and by combining the work done in [10], thus adapting to our case study that concern logistic transport. 


\subsection{Device ontology}

Device ontology describes functionalities, hardware attribute and physical concepts measured by devices: sensor, tags, and actuator. Based on work done in[10][18]and according to the requirement of logistic process we have detected the principles ontology concepts used to better model devices. A representation of the main class related to the device is shown in Figure 2.

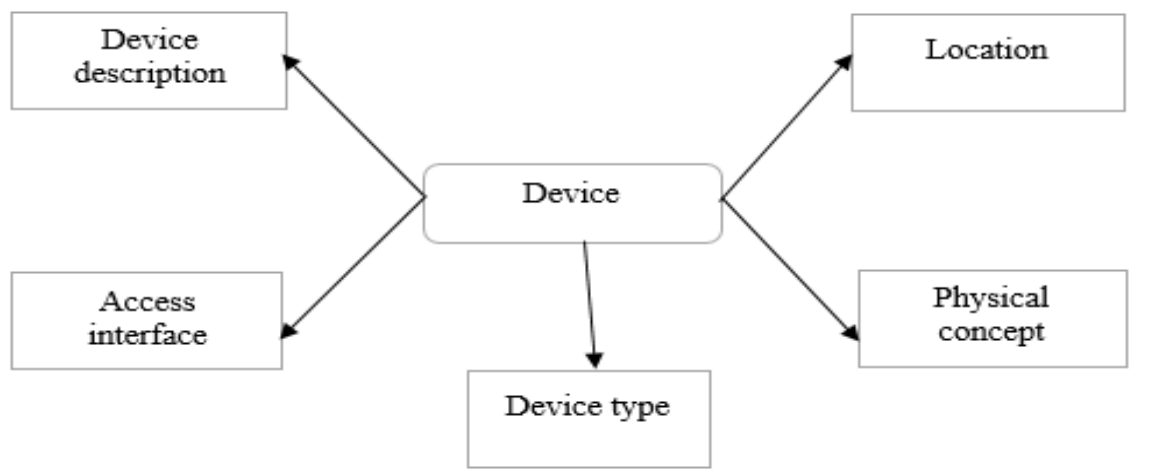

Figure 2: Device and related first class entities

Device type: device can be divided in three main type: sensor, tag, or actuator. When the type is sensor, our ontology will be connected to an external ontology SSN.

Device description: it contains information about device's manufacturer, device's internal hardware components and all information that can be specific to each device, which give a good vision about the quality of device hardware.

Physical concept: it contains real world properties measured by device such as: humidity, temperature, pressure...etc. Thus their value and unit of measurement.

Location: defines the location of the device and it is connected to an external ontology: local and global location ontologies

Access interface: it includes technologies generally used in distributed systems such as: RPC, SOAP, and REST.

\subsection{Thing ontology}

The goods transported during the logistic process have some specific features that need to be taken into consideration, for this reason we have dedicated an ontology especially to model information related to product: their state, location, manufacturer, condition of conservation...etc. The main classes linked to thing concept are grouped in Figure 3. 


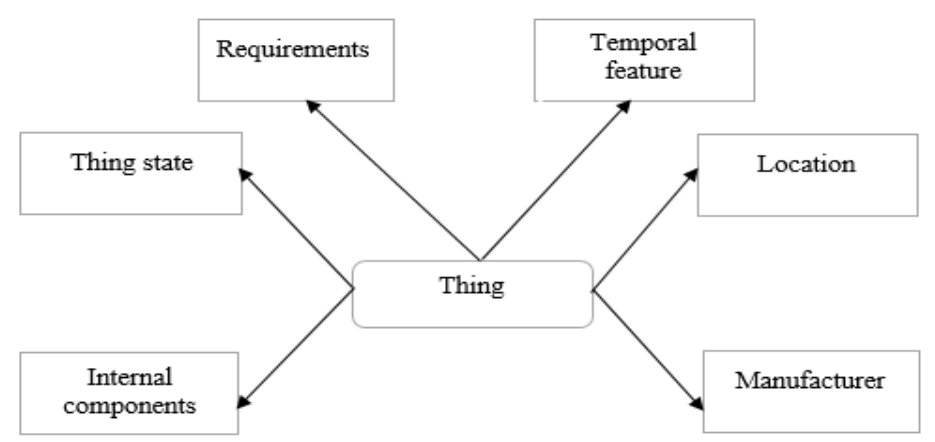

Figure 3: Thing and related first class entities

In fact, manufacturer class contains the historic of product from his point of origin and including all transitional activities to integrate product's component. Internal components regroup the raw materials used for the production of product. Moreover, requirements involve all information needed to transport product in a good conditions such as: high and low temperature allowed, incompatible product, dangerousness constituents. Location tracks the emplacement exact of product and it is similar to the one described in device ontology. Thing state comprises all observable features that we can need to know the state of product such as: temperature, pressure, humidity...etc. Temporal feature specifies the chronologic of product and the way in which his properties are transformed.

\subsection{Logistic ontology}

This ontology is based on core ontology proposed in [14] and it will be extended according to our use case scenario. The most relevant objects for logistic domain are: transport, kind of good, mode of transport, type of equipment used. In addition to these concepts, other logistic situation and events are discussed in [11] and they will be the basis for our ontology. We will describe in more detail in future work this ontology in a logistic transport situation and by using 4PL provider.

\section{Conclusion}

We presented in this paper general middleware architecture for logistic process, based on semantic technologies, especially a set of ontologies that provide interoperability between heterogeneous components and models the different elements of loT involved in the domain of logistic. The relevant aspects are presented by three ontologies: device ontology to describe sensing device and their functionalities, Thing ontology for product and their requirement, and logistic ontology that show most concepts used in the logistic domain.

Our purpose in the future work is to implement the proposed ontologies and evaluate their feasibility, thus handle other part of middleware to improve communication and keep services loosely coupled in order to increase reusability.

\section{REFERENCES}

[1] Z. Sheng, S. Yang, Y. Yu, A. Vasilakos, J. Mccann, et K. Leung, " A survey on the ietf protocol suite for the internet of things: Standards, challenges, and opportunities », IEEE Wirel. Commun., vol. 20, no 6, p. 9198, 2013. 
[2] T. Gruber, « What is an Ontology».

[3] « OWL Web Ontology Language Overview ». https://www.w3.org/TR/owl-features/.

[4] S. Avancha, C. Patel, et A. Joshi, « Ontology-Driven Adaptive Sensor Networks. », in MobiQuitous, 2004, vol. 4, p. 194-202.

[5] D. J. Russomanno, C. Kothari, et O. Thomas, « Sensor ontologies: from shallow to deep models », in System Theory, 2005. SSST'05. Proceedings of the Thirty-Seventh Southeastern Symposium on, 2005, p. 107-112.

[6] «Sensor Model Language (SensorML) | OGC ». http://www.opengeospatial.org/standards/sensorml.

[7] «The Suggested Upper Merged Ontology (SUMO) - Ontology Portal ». http://www.adampease.org/OP/.

[8] " Incubator Report - Semantic Sensor Network Incubator Group ". https://www.w3.org/2005/Incubator/ssn/wiki/Incubator_Report.

[9] C. A. Henson, J. K. Pschorr, A. P. Sheth, et K. Thirunarayan, « SemSOS: Semantic sensor observation service ", in Collaborative Technologies and Systems, 2009. CTS'09. International Symposium on, 2009, p. 44-53.

[10] S. Hachem, T. Teixeira, et V. Issarny, "Ontologies for the internet of things ", in Proceedings of the 8th Middleware Doctoral Symposium, 2011, p. 3.

[11] P. Lian, D.-W. Park, et H.-C. Kwon, « Design of logistics ontology for semantic representing of situation in logistics ", in Digital Media and its Application in Museum \& Heritages, Second Workshop on, 2007, p. 432-437.

[12] J. Leukel et S. Kirn, « A supply chain management approach to logistics ontologies in information systems ", in International Conference on Business Information Systems, 2008, p. 95-105.

[13] X. K. Zhang et T. Tian, "Logistics domain ontology model and its application ", in Advanced Materials Research, 2010, vol. 108, p. 1403-1408.

[14] L. Daniele et L. Ferreira Pires, « An ontological approach to logistics », 2013.

[15] T. Teixeira, S. Hachem, V. Issarny, et N. Georgantas, "Service oriented middleware for the internet of things: a perspective », in European Conference on a Service-Based Internet, 2011, p. 220-229.

[16] S. Bandyopadhyay, M. Sengupta, S. Maiti, et S. Dutta, "Role of middleware for internet of things: A study », Int. J. Comput. Sci. Eng. Surv., vol. 2, no 3, p. 94-105, 2011.

[17] S. Hachem, "Service-Oriented middleware for the large-scale mobile Internet of Things », Université de Versailles-Saint Quentin en Yvelines, 2014.

[18] S. De, P. Barnaghi, M. Bauer, et S. Meissner, « Service modelling for the Internet of Things », in Computer Science and Information Systems (FedCSIS), 2011 Federated Conference on, 2011, p. 949-955. 